\title{
Imaging synchrony
}

\author{
Elias H. Botvinick, MD, ${ }^{a}$ J. William O'Connell, BS, ${ }^{b}$ and Nitish Badhwar, MD $^{c}$
}

\section{See related article, pp. 895-903}

There are 550,000 new cases of heart failure with 1,000,000 hospitalizations and 250,000 heart failure deaths yearly in the USA with similar numbers in the European Union. Cardiac resynchronization therapy (CRT), based in the placement of A-V sequential, biventricular pacemakers, is indicated to treat patients with advanced, medically refractory heart failure, reduced left ventricular (LV) function, and prolonged QRS duration. Normally stimulated in an orderly pattern, conduction is rapid through normal myocardium, bringing near simultaneous, coordinate, and synchronous myocardial contraction. Scarring leads to irregular conduction and varied sequences of dyssynchronous contraction. CRT, seeks to improve mechanical synchrony, but has other benefits. CRT has been shown to improve symptoms and survival, increase exercise tolerance, and quality of life, reduce hospitalization, and bring reverse remodeling ${ }^{1}$ in heart failure patients. Yet, there is now nothing better to determine mechanical synchrony and the potential for CRT improvement, than ECG QRS duration, a crude and imperfect electrical surrogate for mechanical dyssynchrony which excludes many with heart failure and cannot optimize LV pacemaker placement. The current broad inclusion criteria bring variable functional improvement in only $60-70 \%$ of CRT patients. ${ }^{2}$ CRT is invasive, costly, and risky. A better indicator of CRT improvement is needed.

From the Departments of Radiology, Nuclear Medicine Section and Medicine, Cardiovascular Division, ${ }^{a}$ University of California San Francisco, San Francisco, CA; Department of Radiology, Nuclear Medicine Section, ${ }^{\text {b }}$ University of California San Francisco, San Francisco, CA; and Department of Medicine, Cardiovascular Division, ${ }^{\mathrm{c}}$ University of California San Francisco, San Francisco, CA.

Reprint requests: Elias H. Botvinick, MD, Departments of Radiology, Nuclear Medicine Section and Medicine, Cardiovascular Division, University of California San Francisco, San Francisco, CA, USA; botvinicke@medicine.ucsf.edu.

J Nucl Cardiol 2009;16:846-8.

$1071-3581 / \$ 34.00$

Copyright $\odot 2009$ by the The Author(s). This article is published with open access at Springerlink.com.

doi:10.1007/s12350-009-9151-4
Imaging methods present clear potential to evaluate the contraction pattern and measure synchrony. Echocardiography was applied early and widely to evaluate synchrony and CRT. However, the varied echocardiographic synchrony methods are not standardized, are operator dependent, lack reproducibility, and are often lengthy, presenting complex measurements, with limited sampling windows, high noise level, and unproven sensitivity. While small observational studies suggest that echocardiographic synchrony measures can identify CRT "responders" and predict outcomes, their relation to CRT outcomes is irregular with an inability to characterize the contraction abnormality. ${ }^{3}$ A recent multicenter study evaluated 12 popular echocardiographic parameters of dyssynchrony, demonstrating their inconsistency and inability to relate prognosis or track outcome in CRT. ${ }^{4}$ Yet, newer Doppler methods and 3D show renewed promise. ${ }^{5}$

MRI has the potential to express synchrony, and several methods are now evolving. However, the modality is costly, not widely available and is not generally recommended or widely applied to patients with implanted devices.

Scintigraphic methods permit digitization of physiologic and synchrony data. They are highly reproducible, repeatable, and widely available. A count-based method applies first harmonic analysis to extract regional amplitude and phase angle $(\varnothing)$ from the curve-relating myocardial intensity to systolic wall thickening on gated SPECT myocardial perfusion scintigraphy. ${ }^{6}$ The method differentiated paced subjects and those with bundle branch block, from normals with adequate reproducibility, ${ }^{7}$ and demonstrated a sensitivity and specificity of $74 \%$, for the prediction of response to $\mathrm{CRT}^{8}{ }^{8}$ However, data quality may lose accuracy due to undersampling with the conventional 8 frame acquisition and the relationship between wall motion and thickening need not be linear. Regional perfusion could vary with CRT in a manner not proportional to regional function, or outcomes and function may not be measured accurately in underperfused segments.

The mean and standard deviation of $\operatorname{LV} \varnothing(\operatorname{SD} \varnothing)$, derived from first harmonic phase analysis and the phase histogram of the ventricular time activity curve in equilibrium radionuclide angiography (ERNA), have been applied to characterize synchrony. Novel, objective measures of regional contraction and global mechanical 
synchrony, the synchrony $(S)$ and entropy $(E)$ parameters have been developed and applied to planar ERNA as a tool for evaluation and management of HF patients. $S$ expresses the efficiency of contraction within a region of interest (ROI). $S$ can estimate the contraction potential if the ROI were synchronized. $E$ measures the degree of randomness within the ROI, from 0 , with synchronous motion and a single $\varnothing$, to 1 with fully dyssynchronous contraction. It is designed to differentiate among forms of extremely variable regional dyssnchrony. Initially applied in a simulation model, ${ }^{9}$ these parameters were demonstrated to be highly reproducible and repeatable. Compared to SD $\varnothing, S$ and $E$ were better able to differentiate synchrony profiles among a spectrum of patterns of abnormal wall motion. In preliminary clinical protocols normal values were established and these measures were shown to enhance CRT patient selection, to predict and quantitate CRT outcomes, to optimize CRT pacemaker lead placement based on location of the latest contracting segment, to assess synchrony in HF patients with narrow QRS, and to measure RV synchrony. ${ }^{10-16}$

In this issue of the Journal ${ }^{17}$ Wassenaar and coworkers at the Ottawa Heart Institute sought to establish optimal values for image smoothing, histogram noise threshold, and bin size applied in the generation of SD $\varnothing$, $S$, and $E$ in normal subjects and patients with mechanical dyssynchrony. At the optimal imaging values, the sensitivity and specificity for all parameters for detection of mechanical dyssynchrony were very high with inter- and intra-observer correlation coefficients approaching unity. Optimized planar ERNA SD $\varnothing, S$, and $E$, detected mechanical dyssynchrony with low inter- and intraobserver variability.

Our group has a special interest in this manuscript and its findings. After extensive experience in the application of phase image analysis, we developed the $S$ and $E$ parameters and have presented and published evidence of their unique ability and application in CRT patients. ${ }^{9-16}$ The results presented here further optimize and establish the reproducibility of the method and move it forward, closer to clinical application. While certainly not yet proven, the parameters evaluated here have the capability to quantitate synchrony and reproducibly follow it serially. With the phase image from which they are derived, the latest contracting segment can be localized and CRT pacemaker location optimized and the importance of RV synchrony can be evaluated over a spectrum of cardiac pathology. As noted by the authors, further studies assessing the ability of these parameters to predict CRT outcome are required and application of the method to SPECT ERNA could add greater resolution and accuracy.

We have long been aware of the lack of objective, reproducible methods to measure mechanical synchrony, and their need. ${ }^{18}$ Zaret $^{19}$ noted the importance of such synchrony imaging software, and the "obvious ... suitability of [phase ERNA] for the study of patients considered for CRT," stating " "... a more accurate ... definition of those who will benefit [from CRT] is both a clinical and economic necessity." He urged, "Appropriate funding for such studies...." We certainly agree but have already seen that the current economic crisis will stifle innovation and support in this important area of imaging application. ${ }^{20}$ There is every reason to believe that methods such as that evaluated here, could add objectivity and clarity to the selection and implementation of CRT. This could increase its success rate with reduction in the variability of its benefit. If successful, this relatively inexpensive imaging method, or others with such advantages, could more appropriately and effectively apply a costly intervention to a large vulnerable population. This presents great potential benefits of cost-effectiveness, most valuable in this time of economic challenge. As expanded applications of CRT loom, ${ }^{21}$ such an approach may be expected to evolve from an option to a necessity.

\section{Open Access}

This article is distributed under the terms of the Creative Commons Attribution Noncommercial License which permits any noncommercial use, distribution, and reproduction in any medium, provided the original author(s) and source are credited.

\section{References}

1. Bleeker GB, Kaandorp TA, Lamb HJ, Boersma E, Steendijk P, de Roos A, et al. Effect of posterolateral scar tissue on clinical and echocardiographic improvement after cardiac resynchronization therapy. Circulation 2006;113:969-76

2. Cleland JG, Daubert JC, Erdmann E, Freemantle N, Gras D, Kappenberger L, et al. The effect of cardiac resynchronization on morbidity and mortality in heart failure. N Engl J Med 2005; 352:1539-49.

3. Marcus GM, Rose E, Viloria EM, Schafer J, De Marco T, Saxon LA, et al. Septal to posterior wall motion delay fails to predict reverse remodeling or clinical improvement in patients undergoing cardiac resynchronization therapy. J Am Coll Cardiol 2005;46: 2208-14.

4. Conca C, Faletra FF, Miyazaki C, Oh J, Mantovani A, Klersy C, et al. Echocardiographic parameters of mechanical synchrony in healthy individuals. Am J Cardiol 2009;103:136-42.

5. Sogaard P, Egeblad H, Pedersen AK, Kim WY, Kristensen BO, Hansen PS, et al. Sequential versus simultaneous biventricular resynchronization for severe heart failure: Evaluation by tissue Doppler imaging. Circulation 2002;106:2078-84.

6. Brandão SC, Nishioka SA, Giorgi MC, Chen J, Abe R, Filho MM, et al. Cardiac resynchronization therapy evaluated by myocardial scintigraphy with (99m)Tc-MIBI: Changes in left ventricular uptake, dyssynchrony, and function. Eur J Nucl Med Mol Imaging 2009;36:986-96. 
7. Trimble MA, Borges-Neto S, Smallheiser S, Chen J, Honeycutt $\mathrm{EF}$, Shaw LK, et al. Evaluation of left ventricular mechanical dyssynchrony as determined by phase analysis of ECG-gated SPECT myocardial perfusion imaging in patients with left ventricular dysfunction and conduction disturbances. J Nucl Cardiol 2007; 14:298-307.

8. Henneman MM, Chen J, Dibbets-Schneider P, Stokkel MP, Bleeker GB, Ypenburg C, et al. Can LV dyssynchrony as assessed with phase analysis on gated myocardial perfusion SPECT predict response to CRT? J Nucl Med 2007;48:1104-11.

9. O'Connell JW, Schreck C, Moles M, Badwar N, DeMarco T, Olgin J, et al. A unique method by which to quantitate synchrony with equilibrium radionuclide angiography. J Nucl Cardiol 2005; 12:441-50

10. Badhwar N, O'Connell JW, Botvinick EH, Green D. The value of SPECT equilibrium radionuclide angiogram synchrony analysis. J Nucl Med 2008;49:192P (abstr).

11. Badhwar N, O'Connell JW, Botvinick EH, Green D. Equilibrium radionuclide angiogram derived measures of dyssynchrony correlate with clinical outcomes in heart failure patients. J Nucl Med 2008;49:128P (abstr).

12. Badhwar N, O'Connell JW, Botvinick EH, Green D. Utility of ERNA derived measures of dyssynchrony to select heart failure patients for cardiac resynchronization therapy. J Nucl Med 2008;49:274P (abstr).

13. Badhwar N, O'Connell JW, Botvinick EH. Utility of ERNA to guide Coronary Sinus lead placement in Heart Failure patients requiring CRT. CV Young Invest 2nd Prize. J Nucl Med 2006;47:1P (abstr)

14. Badhwar N, Green D, O'Connell JW, Botvinick EH. Evaluation of equilibrium radionuclide angiogram derived measures of interventricular dyssynchrony in heart failure patients requiring cardiac resynchronization therapy. J Nucl Med 2008;49:192P (abstr).

15. Badhwar N, O'Connell JW, Botvinick EH, Green D. Equilibrium radionuclide angiogram derived measures of left ventricular dyssynchrony in heart failure patients with narrow QRS. J Nucl Med 2008;49:192P (abstr).

16. Badhwar N, Johnson C, O'Connell JW, Botvinick EH, Green D. Right ventricular synchrony in patients undergoing cardiac resynchronization therapy (CRT). J Nucl Med 2008;49:190P (abstr).

17. Wassenaar R, O'Connor D, Dej B, Ruddy TD, Birnie D. Optimization and validation of radionuclide angiography phase analysis parameters for quantification of mechanical dyssynchrony. J Nucl Cardiol 2009. doi:10.1007/s12350-009-9119-4.

18. Botvinick, EH. Editorial comment: Scintigraphic blood pool and phase image analysis the optimal tool for the evaluation of resynchronization therapy. J Nucl Cardiol 2004;10:424-8.

19. Zaret Barry L. Cardiac Imaging and CRT: Time to get in phase. J Am Coll Cardiol Imaging 2008;1:614-6.

20. Rae-Dupree J. It's no time to forget about innovation. New York Times, Business Section, p. 1, 2008.

21. Moss AJ, Hall WJ, Cannom DS, Klein H, Brown MW, Daubert JP, et al. Cardiac resynchronization therapy for the prevention of heart failure events. NEJM 2009;361:1056-66. 